\title{
Application of an Equilibrium Model for an Electrified Fluid Interface-Electrospray Using a PDMS Microfluidic Device
}

\author{
Paul R. Chiarot, Sergey I. Gubarenko, Ridha Ben Mrad, and \\ Pierre E. Sullivan
}

Version Post-print/accepted manuscript

Citation Chiarot, Paul R., Sergey I. Gubarenko, Ridha Ben Mrad, and Pierre

(published version) Sullivan. "'Application of an equilibrium model for an electrified fluid interface-Electrospray using a PDMS microfluidic device."'" Journal of Microelectromechanical Systems 17, no. 6 (2008): 1362-1375.

Doi: 10.1109/JMEMS.2008.2006822

How to cite TSpace items

Always cite the published version, so the author(s) will receive recognition through services that track citation counts, e.g. Scopus. If you need to cite the page number of the author manuscript from TSpace because you cannot access the published version, then cite the TSpace version in addition to the published version using the permanent URI (handle) found on the record page.

This article was made openly accessible by $U$ of 'T Faculty.

Please tell us how this access benefits you. Your story matters. 


\title{
APPLICATION OF AN EQUILIBRIUM MODEL FOR AN ELECTRIFIED FLUID INTERFACE - ELECTROSPRAY USING A PDMS MICROFLUIDIC DEVICE
}

\author{
Paul R. Chiarot Sergey I. Gubarenko Ridha Ben Mrad Pierre E. Sullivan \\ University of Toronto \\ Department of Mechanical and Industrial Engineering \\ 5 King's College Road \\ Toronto, Ontario, Canada, M5S 3G8 \\ Phone: 416-978-3110 Fax: 416-978-3453 Email: sullivan@mie.utoronto.ca
}

\begin{abstract}
An experimental investigation of an electrified fluid interface is presented. The experimental findings are related to a previously developed analytical model used to determine when a fluidic interface under electrical stress is in equilibrium (Phys. Fluids, 20(4), 043601) and to observations reported in the literature. The effect of key parameters on causing the interface to rupture, form, and maintain an electrospray is investigated. The experimental results reveal the dependence of interface shape on operational parameters, the impact of the interface apex angle on equilibrium, the conditions that cause either dripping mode or cone-jet mode, and the structure of operational domains. This study confirms predictions made using the analytical model, including the range of parameters that cause the onset and steadiness of a quasi equilibrium (electrospray) state of the interface. Testing is performed using an electrospray emitter chip fabricated from two layers of Polydimethylsiloxane (PDMS) and one layer of glass. The model and experimental results assist in design decisions for electrospray emitters. Applications of electrified interfaces (electrosprays) are found in mass spectrometry, microfluidics, material deposition, and colloidal thrusters for propulsion.
\end{abstract}

\section{INTRODUCTION}

Electrospray can be found in a number of different areas, including material deposition [1-2], colloid thrusters for propulsion [3], and most notably biological analysis. Fenn et al.[4] used the electrospray process to transfer biomolecules from solution into the gas phase for the purpose of mass spectrometry analysis. Through the formation of a Taylor cone, the biomolecules are ejected from the apex of the cone into the gas phase. Recently, there has been considerable interest in developing microscale electrospray emitters for use in mass spectrometry [5-8]. Microscale emitters have the advantage of improved sample handling and allow for the possibility of combining additional processes on chip. For a thorough review of microfluidic systems coupled to mass spectrometry via electrospray, see Koster and Verpoorte [9]. 
In a recent study, Gubarenko et al. [10] developed a model of an electrified fluid interface that examined the impact of operational parameters on interface equilibrium. The model predicted the conditions that cause the interface to rupture and form an electrospray and the conditions necessary to maintain an electrospray once it is formed. Details relating to interface shape and generatrix were also predicted. The purpose of the current work is to apply the model to an experimental study of an electrified interface to account for the observed phenomena and to reveal the relationship between operational conditions and interface properties - most notably during electrospray. As part of this study, an electrospray emitter chip was developed to manipulate an electrified interface and generate electrospray.

Zeleny [11] and Taylor [12] report some of the earliest work on the topic of electrified interfaces. Taylor proposed a concise analytical model for the formation and structure of the observed electrified cone: the 'Taylor cone'. He reported an equilibrium expression for the electrified cone and calculated only one possible angle where equilibrium exists. The emission of the charged droplets from the apex of the electrified cone is an electrospray (Figure 1).

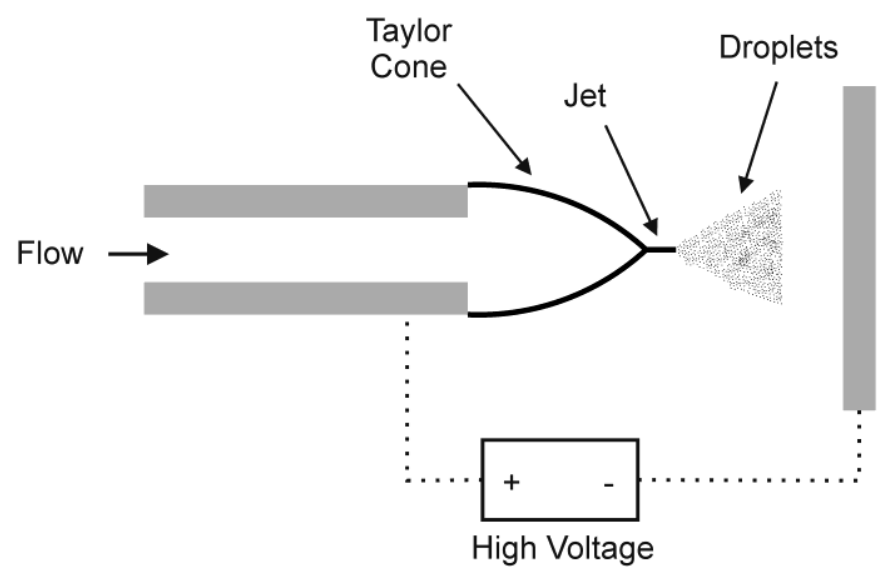

Figure 1 - Formation of an electrospray. The application of a large electric potential to an air-fluid interface causes the interface to deform and emit a spray of charged droplets. The interface is 'electrified'.

More recently, a variety of models have been proposed that describe an electrified interface and the onset of jetting. Studies into the shape, stability, and structure of an electrified interface have been made by Basaran and Scriven [13], Harris and Basaran [14], Wohlhuter and Basaran [15], Stone et al. [16], and Cherney [17]. Sujatha et al. [18] used the variational principle to describe the equilibrium of the electrified interface, and noted the lack of an excess pressure term in Taylor's equilibrium model. Reznik et al. [19] investigated the shape evolution of an electrified interface over time studying isolated droplets and the impact of electrical bond number on interface evolution. 
Experimental evidence has revealed a variety of cone angles are possible, and this deviation from Taylor's predicted angle was addressed by Fernandez de la Mora [20], who accounted for the effect of space charge in the emitted jet on the cone angle. Fernandez de la Mora and Loscertales [21] and GananCalvo et al.[22] report scaling laws to predict spray current and emitted droplet size of a conical electrified interface. Cloupeau and Prunet-Foch [23] identified several distinct 'modes' or shapes that the interface can take when under electric stress. Modes identified by Cloupeau and Prunet-Foch are commonly employed throughout the literature and in this study. A recent thorough review of past work on the subject of Taylor cones is provided by Fernandez de la Mora [24].

The equilibrium model [10] addressed limitations found in previous models of an electrified interface. Using the concept of an operational domain, the model identified the conditions necessary to cause an interface to rupture and form an electrospray and the conditions necessary to maintain an electrospray. The model highlighted the importance of the apex angle on interface equilibrium and predicted varying configurations for the cone generatrix. An experimental study was necessary to demonstrate the quality of this model and to show how its results can be applied to manipulate an electrified interface and predict equilibrium states of the interface. The results of the experimental study, coupled with the equilibrium model, can also be used to explain observed phenomena and give insight into the behavior of an electrified interface and the electrospray mechanism.

The electrospray emitter chip developed in this study is compatible with traditional microfluidic device fabrication and is demonstrated to be compatible with on-chip sample processing. This design is less complicated to fabricate compared to other concepts, and the fact that it is a closed system means it is less susceptible to solvent evaporation and channel contamination compared to other open channel emitters [7][25]. In this study, the chip is used to evaluate the properties of an electrified interface.

The ability to predict the conditions necessary to form and maintain an electrospray allows improved design decision to be made for microscale electrospray emitters used in the applications identified above. It is useful to apply only the minimum required electric field (a function of voltage and electrode separation distance) and pressure difference to form and maintain an electrospray, and to avoid excessively large voltages and pressures. This will minimize the power requirements, the geometric constraints on channel size, and the mechanical stresses on the emitter device. 


\section{Electrospray EMITTER CHIP FABRICATION}

The electrospray emitter chip fabricated in this study is an enclosed system whose primary structure is made of two layers of Polydimethylsiloxane (PDMS) (Dow Corning) and one layer of glass. One of the PDMS layers is the 'channel layer' and the other is the 'intermediate layer'. The electrospray is formed from the end of metal tubing inserted into the PDMS at the end of an upstream channel network. PDMS is a useful material for the production of microfluidic systems and has been successfully used in the literature [26-27].

The channel layer is a network of microfluidic channels that can be used for upstream processing of the fluid undergoing electrospray. The design of the electrospray emitter chip allows it to be a platform where any desired upstream process could be combined with electrospray. To facilitate this, it is possible to modify the geometry of the channel layer to fit the needs of the upstream process. A metal layer is also incorporated that can be patterned into any desired shape to serve as an electrical layer. The intermediate layer is situated between the glass layer and channel layer and is used to properly position the metal tubing and to prevent leakage from around the tubing. A possible upstream process that can be incorporated is capillary electrophoresis (CE)[28]. CE would incorporate the use of the electrical (metal) layer and two separate channel networks; one for the separation and one for the supporting sheath flow.

The fabrication procedure starts by cutting the glass substrate to the appropriate size and then drilling a 2 millimeter hole for fluidic access. The glass substrate is then cleaned in a hot Piranha (3:1 $\mathrm{H}_{2} \mathrm{SO}_{4}: \mathrm{H}_{2} \mathrm{O}_{2}$ ) solution for 10 minutes. A metal layer is deposited on top of the glass layer and used for upstream processing of the sample. Metal (chromium or gold/chromium) can be evaporated to a thickness of $400 \mathrm{~nm}$ and patterned to the desired shape using standard techniques.

The internal geometry of the PDMS emitter is formed by making a negative relief of the reservoir and channel network. The negative relief is made of patterned SU8 (Microchem) on a silicon wafer substrate. First, the silicon wafer is cleaned in a hot Piranha solution for 10 minutes and then a dilute hydrofluoric acid solution (10:1 HF) for 5 minutes. SU8 2100 is spun (500rpm / 88rpms ${ }^{-1} / 20$ s to spread and 2000rpm / 264rpms ${ }^{-1} / 50$ s to coat) on to the wafer to create a thickness of $140 \mu \mathrm{m}$, however spin speeds can be controlled to create a range of thickness. The wafer is baked for $5 \mathrm{mins}$ at $65^{\circ} \mathrm{C}$ and 35 mins at $90^{\circ} \mathrm{C}$ and then exposed to UV light using a mask aligner (Karl Suss) to transfer the desired pattern. The wafer is again baked for 15 minutes at $90^{\circ} \mathrm{C}$ and developed in SU8 developer leaving only the desired pattern. This pattern will be used to form the channel network in the PDMS. The emitter in this design uses channel networks with widths of 150 - $300 \mu \mathrm{m}$ and a height of $140 \mu \mathrm{m}$. 
P. R. Chiarot, S. I. Gubarenko, R. Ben Mrad, and P. Sullivan, “Application of an Equilibrium Model for an Electrified Fluid InterfaceElectrospray Using a PDMS Microfluidic Device,” J. Microelectromechanical Syst., vol. 17, no. 6, pp. 1362-1375, 2008.

10.1109/JMEMS.2008.2006822

a)

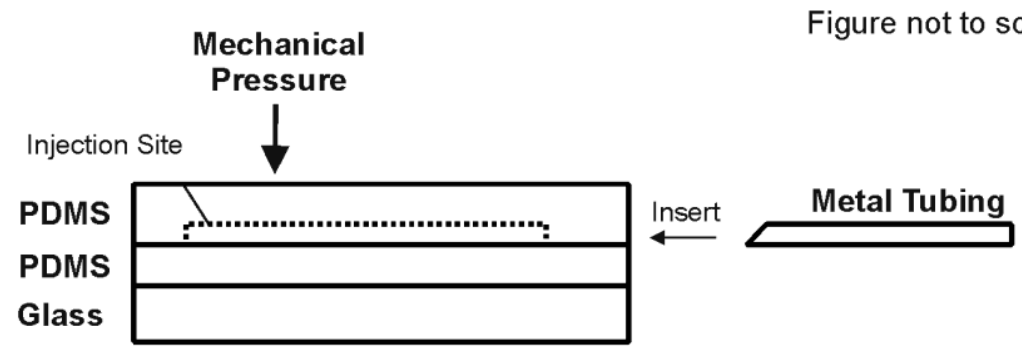

b)

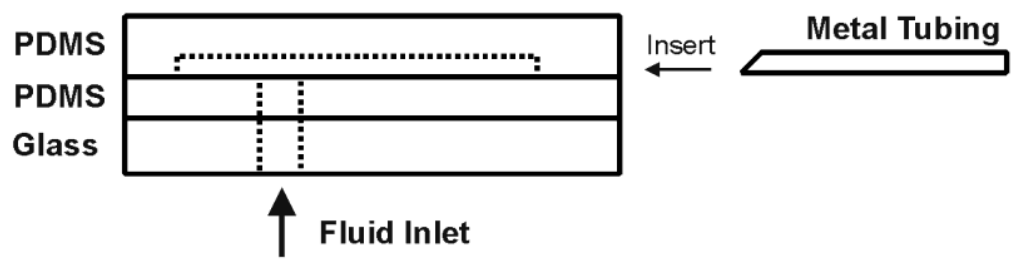

c)

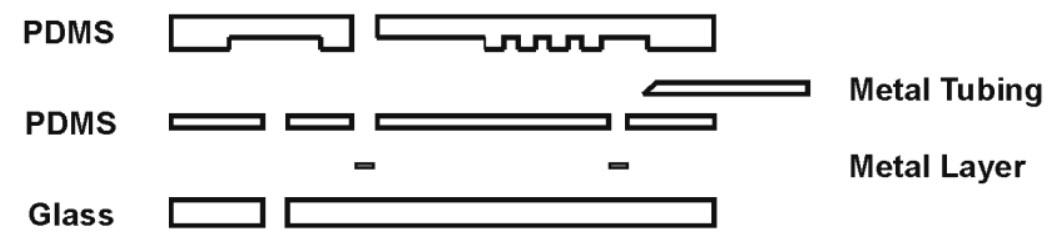

Nanoport \ए

d)

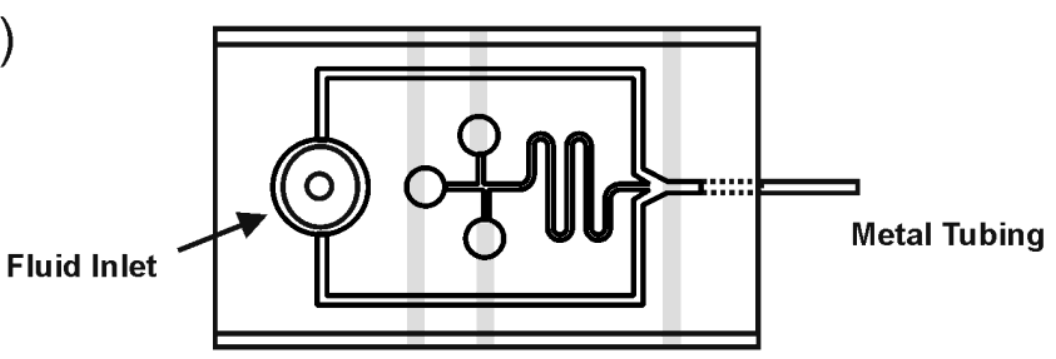

Figure 2 - Planar views of the emitter prototype. The prototype is composed of 2 layers of PDMS and 1 layer of glass. Metal tubing is inserted into the PDMS up to the end of the channel network. The electrospray is formed at the end of the tubing. a) (simplified cross sectional view) a sample is injected into a reservoir where it is briefly stored and mechanical pressure is applied to the surface of the PDMS to generate pressure b) (simplified cross sectional view) a through hole allows pressure to be applied via a syringe pump c) a detailed cross sectional view of the emitter incorporating a CE channel network d) a top view of the emitter with $\mathrm{CE}$ channel network.

PDMS is prepared by mixing the polymer solution with the curing agent in a ratio of 10:1. The mixed PDMS is then poured over the SU8 relief structure and silicon wafer that is stored in a Petri dish (this will be the channel layer) and into a flat Petri dish containing no wafer (this will be the intermediate layer). The mixed solution is then placed in a vacuum chamber for 30 minutes to remove any air bubbles 
trapped in the mixture. The PDMS filled dishes are then transferred to a convection oven at $80^{\circ} \mathrm{C}$ for 2.5 hours. The thickness of the PDMS layers can be controlled by carefully measuring the dispensed mass of the polymer solution and curing agent. The emitter chip in this study has a channel layer that is $\sim 1-2 \mathrm{~mm}$ thick and an intermediate layer that is $\sim 200 \mu \mathrm{m}$ thick. The intermediate layer has holes for access to the metal layer on the surface of the glass wafer.

After curing, the PDMS layers are peeled off the silicon wafer / SU8 relief structure and the flat Petri dish. The relief structure has now been formed in the channel layer of the PDMS. The channel layer and the intermediate layer are cut into rectangular sections of equivalent size and through holes are punched as required. The three layers are bonded together by exposing the bonding surfaces to an oxygen plasma at $65 \mathrm{mT}$ and $70 \mathrm{~W}$ for 15 seconds using an RIE/ICP etcher. After plasma exposure, the intermediate layer is aligned with the glass substrate and the two surfaces are contacted. Alignment is assisted by coating the layers with methanol so that they can move relative to each other. Once the layers are properly aligned, the sample is placed on a hotplate to evaporate the methanol, and a bond is spontaneously formed. The same bonding procedure is used to align and bond the channel layer and the intermediate layer. An enclosed channel network has now been formed. A simplified cross sectional layout of the prototype emitter is shown in Figure $2 a$ and Figure $2 b$ and a detailed cross section of the emitter incorporating CE is shown in Figure 2c. A top view of the device is shown in Figure $2 \mathrm{~d}$.

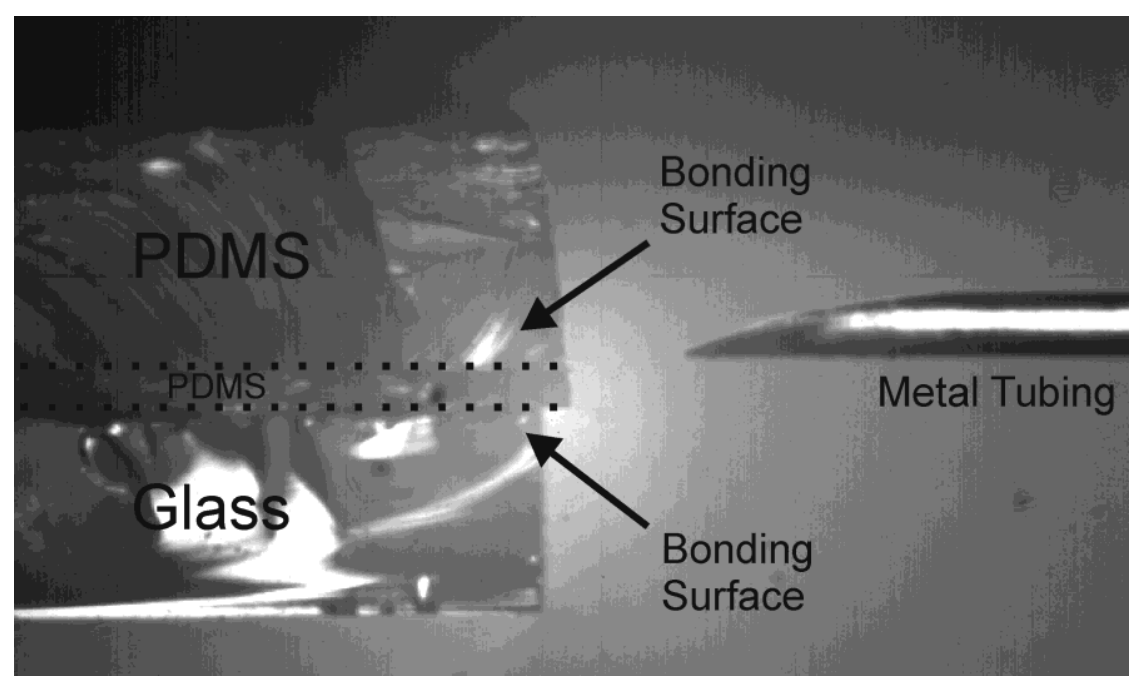

Figure 3 - Alignment of the metal tubing. The metal tubing is aligned to the bonding surface between the PDMS layers and inserted using a precision stage.

A critical step in the fabrication procedure is the alignment and positioning of the metal tubing (Figure 2). The tubing is where electrical connections are made and the electrospray is formed from its 
edge. For this study, tubing with an ID of $140 \mu \mathrm{m}$ and an OD of $300 \mu \mathrm{m}$ is used. The metal tubing is aligned with the top of the intermediate layer (Figure 3) and with the edge of the channel using a microscope. The tubing has a sharp edge than can easily penetrate PDMS. Using a mechanical stage, the emitter chip is slowly advanced until the metal tubing contacts the edge of the channel. The correct positioning is checked again visually using a microscope.

The compliance of the PDMS ensures that the tubing is held firmly in place and that no leakage occurs around the edge. Using the intermediate layer, the tubing mates to the center of the enclosed channel and is completely surrounded by PDMS. Testing showed that it is necessary to have the intermediate layer to improve the seal around the tubing and prevent leakage. To ensure a tight seal, the edge of the emitter chip is clamped overnight in the vicinity of the tubing. At the low flow rates used in this study (on the order of $\sim 1 \mu \mathrm{L} / \mathrm{min}$ ), no leakage occurred around the edge of the tubing. The compliance of the PDMS also helps to reduce the formation of dead volumes that often occur for similar concepts at the end of a channel network.

The final step in the fabrication of the emitter chip is to evaporate a layer of parylene over the entire device. A parylene coater (Specialty Coating Systems) is used to coat the device - and most importantly the metal tubing - with a parylene layer 1-2 $\mu \mathrm{m}$ thick. In other emitter designs, liquid at the electrospray orifice would tend to spread out, negatively affecting the stability of the Taylor cone. This was particularly a problem for flat edge emitters [5] where the liquid to be sprayed would tend to wet the surface adjacent to the orifice. Surface coatings have been applied to address this problem [29]. In this study, the hydrophobicity of the parylene and pinning of the contact line at the parameter of the metal tube ensures that the droplet / Taylor cone is well isolated at the edge of the metal tubing. A small section of parylene is removed from a portion of the metal tube close to the PDMS layers (i.e. away from the end of the tubing where the Taylor cone is formed) using a sharp blade so that electrical connections can be made. Completed prototypes are shown in Figure 4. 


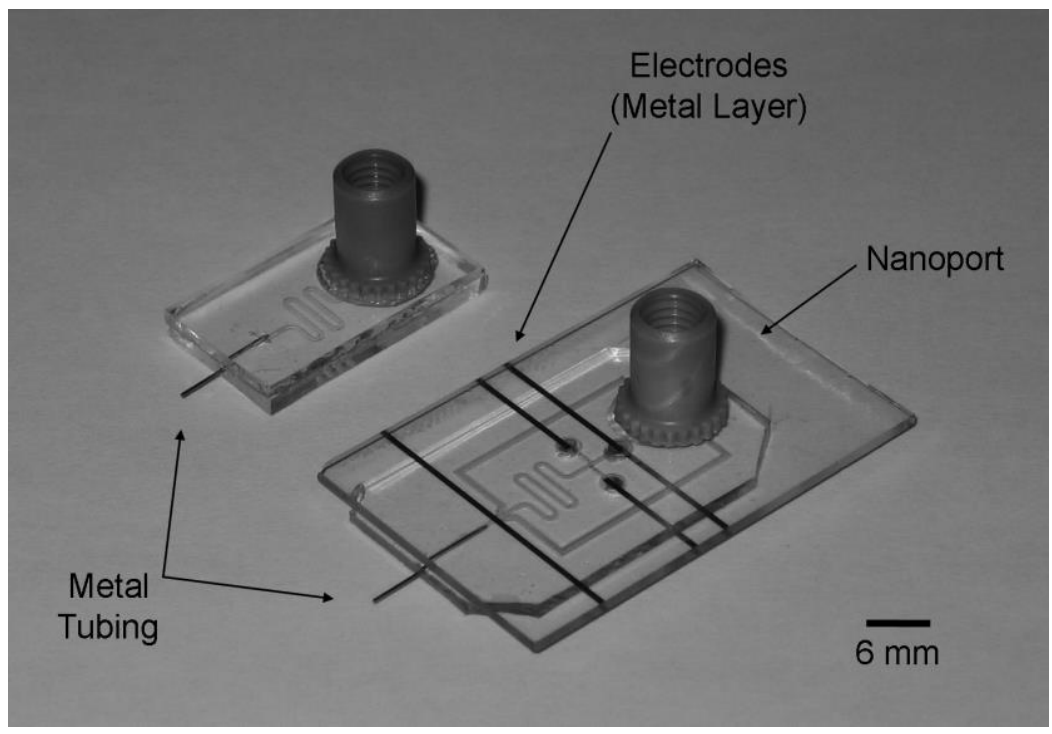

Figure 4 - Examples of the emitter prototypes used in this study. The prototype on the right has electrodes (a patterned metal layer) that can be used in CE. The prototypes use a Nanoport for external fluidic connections to the syringe pump. The channel networks for the prototypes are filled with dye so they can be seen in the figure. The prototypes are shown upside down, as they are typically operated with the Nanoport pointing down and the through holes in the channel layer pointing up.

Fluid flow and pressure can be supplied to the emitter using a syringe pump (Figure 2b), where the connection is made with a Nanoport (Upchurch Scientific). This method is useful for characterizing the performance of the emitter and evaluating the equilibrium model - and it is used in this study. An alternate approach is using mechanical pressure (Figure 2a) supplied by a clamp whose separation can be accurately controlled. In this approach, a hole is not drilled in the glass layer. The sample to be electrosprayed is injected into a reservoir chamber using a small gauge needle. The compliance of the PDMS seals the hole after the needle is removed, preventing leakage (the hole can also be covered with epoxy). Pressure from the clamp deflects the PDMS over the reservoir and forces the fluid into the channel network and towards the end of the metal tube. Using this approach, a stable electrospray can be formed for a short duration of time.

The main advantages of this design is it is compatible and easily integrated with other microfluidic components used in upstream processing, it is uncomplicated to fabricate, it has limited dead volumes, and since it is a closed system, it is not susceptible to solvent evaporation and channel contamination.

In this study, the prototype is used to examine the properties of an electrified fluid interface. The investigation will focus on the operating conditions necessary to cause an interface to rupture and form an electrospray, the conditions necessary to maintain an electrospray once it is formed, and issues related to 
the shape, equilibrium, and operational domain of an electrified interface. The results are connected to the previously developed equilibrium model.

\section{EXPERIMENTAL DETAILS}

\section{Materials and Setup}

In all cases, the bulk fluid used in this study is a $100 \mu \mathrm{M}$ solution of Rhodamine B in $70: 30$ $\mathrm{MeOH}: \mathrm{H}_{2} \mathrm{O}$ solution with $1 \% \mathrm{AcOH}$ or a $100 \mu \mathrm{M}$ solution of Rhodamine B in $85: 15 \mathrm{MeOH}: \mathrm{H}_{2} \mathrm{O}$ solution with $1 \% \mathrm{AcOH}$. These solutions were selected because they are common solvents and are similar to the solutions that were used to test the microscale emitters reported above. The surface tension for the 70:30 $\mathrm{MeOH}: \mathrm{H}_{2} \mathrm{O}$ solution is $27.48 \mathrm{mN} / \mathrm{m}$ and for the $85: 15 \mathrm{MeOH}: \mathrm{H}_{2} \mathrm{O}$ solution is $24.74 \mathrm{mN} / \mathrm{m}$ [30]. Rhodamine $\mathrm{B}$ is a fluorescent dye and is used to improve the recording, analysis, and display of the interface.

The flow rate was controlled using a syringe pump (Cole Parmer) and the interface was observed and recorded using an inverted fluorescent microscope (Leica) and CCD camera (Sony). Using optical filters and a dichromatic mirror (a filter cube), the microscope can illuminate the interface with light at a wavelength of 515-560 $\mathrm{nm}$ and pass light at a wavelength exceeding $590 \mathrm{~nm}$ to the CCD camera. These wavelengths are ideal for the excitation / emission spectrum of Rhodamine B. Voltage is applied using a high voltage source (Labsmith HVS448) with an operating range of $0-3000 \mathrm{~V}$. The radius at the base of the interface in all of the captured images is $150 \mu \mathrm{m}$, which is the same as the outer radius of the metal tubing.

Images of the interface are captured at a resolution of 640x480 in PNG format and processed using MATLAB. Custom code was written to find the interface and describe it as points in an x-y plane or as a polynomial function with up to third order accuracy.

\section{Imaging of Interface and Jet}

The use of Rhodamine B allows for the interface to be more easily visualized and processed. However, it is typically found that the emitted jet can not be visualized when illuminated by the filtered light. This is likely because of the narrow bandwidth that is applied and the small volume of the jet. The jet can be visualized when the full spectrum (bandwidth) of light is applied. 
This is demonstrated for the interfaces shown in Figure 5 and Figure 6. Figure 5a and Figure $6 a$ both shows an interface under full spectrum light and the emitted jet can easily be seen. Figure 5b and Figure $6 \mathrm{~b}$ show the same interface and jet but this time illuminated with filtered light $(515 \mathrm{~nm}-560 \mathrm{~nm})$. The jet can no longer be seen but the boundary of the interface is clearly defined. Figure $5 \mathrm{c}$ and Figure 6c show an image processed in MATLAB where the interface is described as a polynomial with $3^{\text {rd }}$ order accuracy or as points in the $\mathrm{x}-\mathrm{y}$ plane. In all cases, the function describing an entire interface is fitted to the hundreds of pixels that are located at the interface. When describing an entire interface as $x-y$ points, normally only 5-10 points are taken. The interface is axis-symmetric and only half needs to be considered. All frames are aligned so that the bottom of the image is aligned with the end of the metal tubing. This is confirmed visually and simplifies image processing.
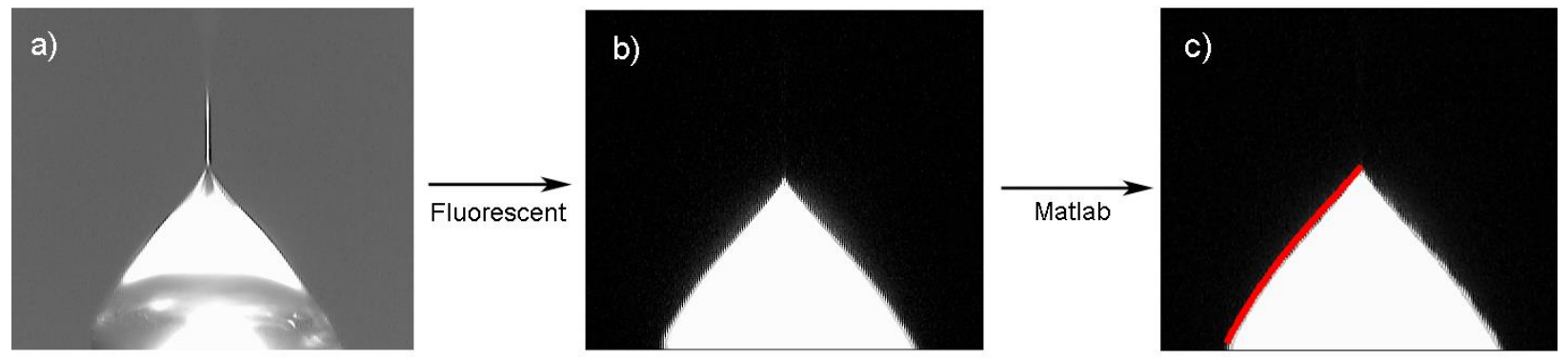

Figure 5 - Image of an electrified interface emitting an electrospray. The fluid is $100 \mu \mathrm{M}$ solution of Rhodamine $\mathrm{B}$ in 70:30 MeOH: $\mathrm{H}_{2} \mathrm{O}$ solution with $1 \% \mathrm{AcOH}$. The radius at the base of the interface is $150 \mu \mathrm{m}$. a) An image in full spectrum light where the emitted jet can be seen b) Image in filtered light c) Image processed in MATLAB where a $3^{\text {rd }}$ order fit to the entire interface is shown.
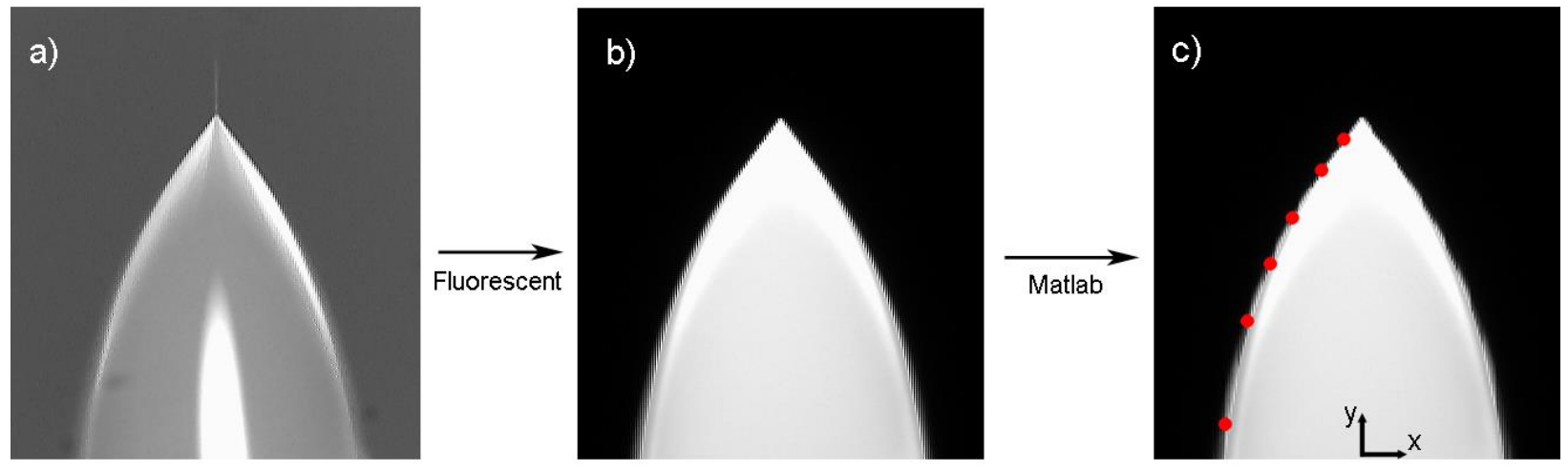

Figure 6 - Image of an electrified interface emitting an electrospray. The fluid is $100 \mu \mathrm{M}$ solution of Rhodamine $\mathrm{B}$ in

70:30 MeOH: $\mathrm{H}_{2} \mathrm{O}$ solution with $1 \%$ АcOH. The radius at the base of the interface is $150 \mu \mathrm{m}$. a) Image in full spectrum light where the emitted jet can be seen b) Image in filtered light c) Image processed in MATLAB where points in the $x-y$ plane at the location of the interface is shown. 
In this study, since we are most concerned with interface shape and equilibrium, only the fluorescent images of the interface are analyzed. The existence of the emitted jet and spray is visually verified by viewing it through the microscope or directly.

\section{THE EQUILIBRIUM MODEL}

For a full description on the development of the equilibrium model, the reader should consult Gubarenko et al. [10]. The model defines the 'critical function':

$$
\psi_{s}(x, \mathbf{z}) \equiv \sin \theta_{s}(x, \mathbf{z})=s-k p x+\kappa \tau_{s}(x, \mathbf{z})
$$

In Eq. $1, x$ is the coordinate along the width of the interface, $\theta$ is the interface angle, $s$ is the sine of the interface angle at the apex, $p$ is the pressure difference, $\tau_{s}$ is the integral of electric (Maxwell) stress $\left(T_{n n}\right)$ along the interface, and $\mathbf{z}$ is a ( $3 \times 1)$ vector of the operational parameters (pressure, voltage, and electrode separation distance). The notation $\theta_{s}$ indicates that the interface angle is a function of the sine of the apex angle. $\kappa$ and $k$ are non-dimensional parameters that compare the importance of electrical stress to capillary stress and pressure to capillary stress, respectively. The dimensional separation distance between the interface and counter electrode is defined as $\bar{h}$. The modeling domain is shown in Figure 7 and shows the dimensional notation (Eq. 1 is shown in non-dimensional form). It is important to note that the apex angle (interface angle $\theta$ at $x=0$ ) and classic Taylor angle $\theta_{\mathrm{T}}$ are related as $\theta+\theta_{\mathrm{T}}=90^{\circ}$. In Figure $7, L_{\varepsilon}$ is the characteristic length, $\bar{T}_{n n}$ is the electrical stress normal to the interface, $\theta$ is the interface angle, $\gamma$ is the surface tension coefficient, $\mathbf{n}$ is the unit normal to the interface, $\bar{p}_{f}$ is the fluid pressure, and $\bar{p}_{a}$ is the reference (atmospheric) pressure. The curvature of the interface is defined as the divergence of the normal along the interface $(\bar{\nabla} \cdot \mathbf{n})$. 


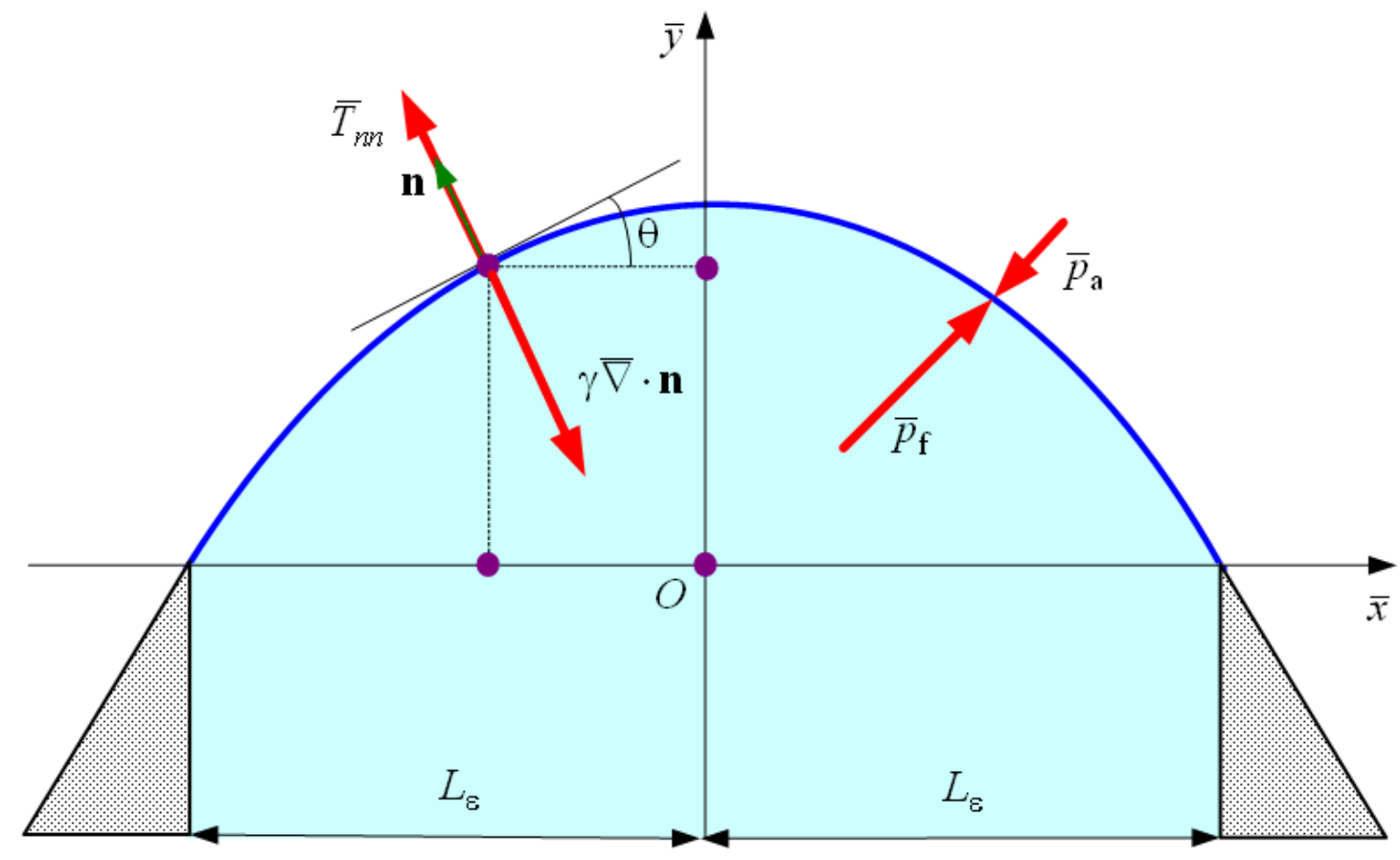

Figure 7 - Modeling Domain. The bars over the quantities represent dimensional notation.

The critical function is shown to be a maximum at $x=-1$. Thus, the critical function determines a 'critical surface' in the 3D domain of operational parameters where $\psi_{s}(-1, \mathbf{z})=1$. Fixing any one of the operational parameters allows for a 'critical curve' to be expressed in two-dimensional space. Equilibrium exists if the parameter values are set such that the critical function $\psi_{s}(-1, \mathbf{z})<1$ (note that the definitions of 'equilibrium' are given in the following subsection). The critical surface represents the boundary of an equilibrium state and the transition from one equilibrium state to another. There exist two types of critical surfaces: critical surface $\psi_{0}(\mathbf{z}) \equiv\left[\psi_{s}(x, \mathbf{z})\right]_{s=0, x=-1}=1$ for parameter $s=0$ determines the 'equilibrium' subdomain in the operational domain and critical surface $\psi_{*}(\mathbf{z}) \equiv\left[\psi_{s}(x, \mathbf{z})\right]_{s=s_{*}, x=-1}=1$ for parameter $s=s_{*} \neq 0$ determines the 'quasi equilibrium' subdomain in the operational domain. Quasi equilibrium implies that a narrow jet of liquid (an electrospray) is emitted from the apex of the interface.

The critical curve separates the operational domain into two subdomains where equilibrium (both static and quasi) can and can not exist. The line is where the critical function from the equilibrium model is equal to 1. In this study, the parameter that is typically fixed is electrode separation so that the impact of applied voltage and pressure can be focused on.

The sine of the interface angle at the middle of the interface $(s)$ is an important term. As noted by Eggers [31], treatment of the interface near the cone tip (apex) is difficult and many past schemes have 
been unreliable. Treatment of the apex angle in the model used here avoids these problems. When $s=0$, the line dividing the domains is considered the transition from static equilibrium to quasi equilibrium. When $s \neq 0$, the line dividing the domains represents the transition from quasi equilibrium to nonequilibrium. It is useful to plot the critical function equal to 1 for several values of $s \neq 0$ to visualize how the apex angle affects the available range of operating parameters for the quasi equilibrium state.

Two important items that the equilibrium model addresses are (a) the concept of static equilibrium, quasi equilibrium and the apex angle term, and (b) the pressure difference across the interface. These items must be defined for the experimental study, and are discussed in the following subsections.

\section{Static Equilibrium, Quasi Equilibrium, and the Apex Angle Term}

Static equilibrium implies that all forces are in balance on the interface, the angle at the middle of the interface (the apex angle) is zero degrees, and the interface is not emitting a spray. In the equilibrium model, this situation is the equivalent of the critical function for $s=0$ having a value less than 1 . An interface in static equilibrium is shown in Figure 8. Figure 8a shows the interface under only pressure and surface tensions forces, and Figure $8 \mathrm{~b}$ shows the same interface $100 \mathrm{~ms}$ later under pressure, surface tension, and electric stress.
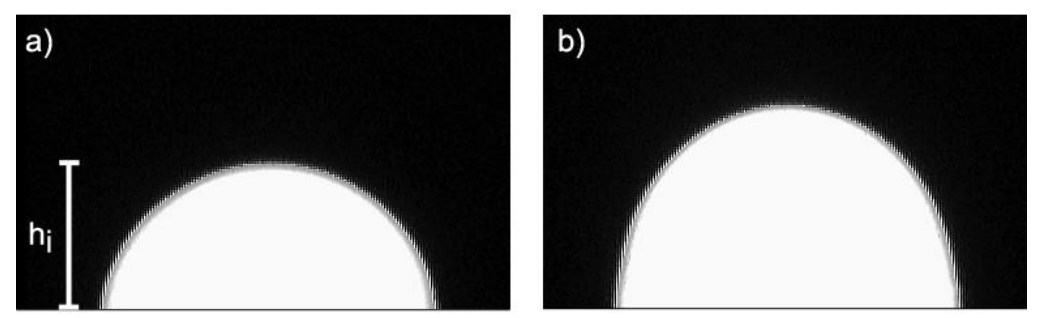

Figure 8 - Images of an interface in static equilibrium. a) The interface under only pressure and surface tension forces. The maximum height of the interface is identified as $h_{i}$. b) The same interface $100 \mathrm{~ms}$ later but now under electrical stress - the interface is now elongated.

When the term quasi equilibrium is applied, an assumption is made that all forces are in equilibrium on the interface. This assumption breaks down very close to the apex of the cone since the fluid is in motion. However, this assumption can be considered valid at all points away from the apex of the interface. Quasi equilibrium indicates that the angle at the apex is not equal to zero $(s \neq 0)$, the critical function (for $s \neq 0$ ) has a value less than 1 , and that the interface is emitting an electrospray. Examples of quasi equilibrium states for the interface are shown in Figure 9a - 9d. 

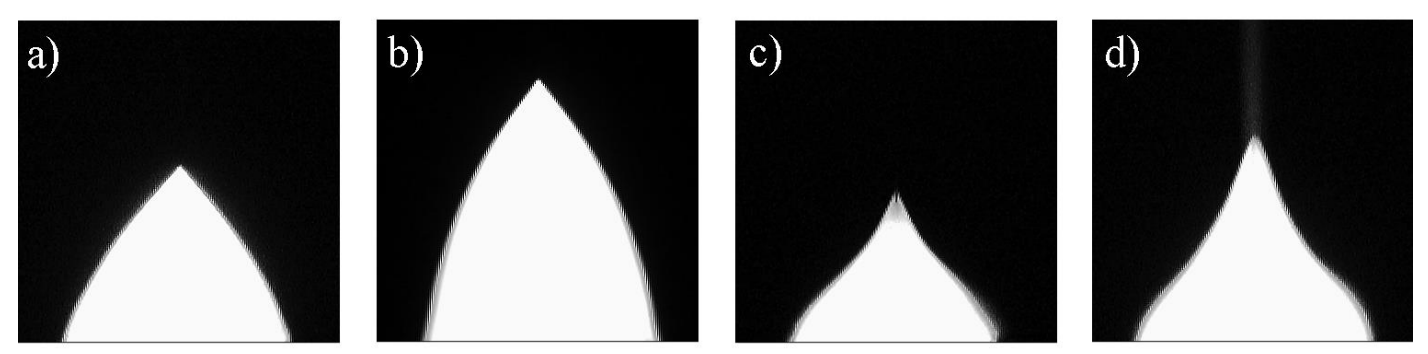

Figure 9 - Images of quasi equilibrium states for the interface. In all cases, the interface is emitting a spray.

These classifications of equilibrium, both static and quasi, have been verified through the experimental work performed in this study. In all measurements, no angle other than zero could exist at the middle of the interface when the whole interface is in static equilibrium. In all cases, if a non-zero angle was found to exist at the middle of the interface, the interface was emitting a spray and in quasi equilibrium.

To solve for the critical function when the interface is in a quasi equilibrium mode, and to understand how apex angle affects equilibrium, it is necessary to know the value of the apex angle term. When developing the equilibrium model, Gubarenko et al. investigated minimizing the potential energy for all forces applied to the interface to solve for an analytical expression for this term [10]. In this study, the apex angle term is calculated from the experimental data by fitting a linear function to several points (on the order of 10 to 20 ) to a portion of the interface in the vicinity of the apex using MATLAB as shown in Figure 6.

\section{Pressure Difference Across the Interface}

Usage of the equilibrium model and critical function means the pressure difference across the interface must be known. This pressure dictates the location of the operating point of the interface in the operational domain. There is no reasonable way to directly measure this pressure; therefore, it is determined indirectly. The pressure difference across the interface subject to only pressure and surface tension forces can be determined if the curvature is known; a fact that is well understood using the YoungLaplace equation. This pressure difference is related to the amount of fluid contained by the interface, which is controlled using the syringe pump. In this study, a 'positive' pressure difference refers to a higher pressure in the fluid compared to the atmosphere and the magnitude of the pressure in the fluid is expressed relative to atmospheric pressure. 
When the electric forces are equal to zero, it is possible to use the equilibrium model to show that the interface will be a spherical cap, and this result is to be expected. The interface can be considered as a portion of a circle with radius $1 / k p$ and center located at $-\sqrt{1-k^{2} p^{2}} / k p$. Using this result it is possible to relate the pressure difference for an interface subjected to pressure and surface tension forces to the non-dimensional height $\left(\mathrm{h}_{\mathrm{i}}\right)$, depicted in Figure $8 \mathrm{a}$, and the non-dimensional quantity $k$ :

$$
p=\frac{2 h_{i}}{k\left(1+h_{i}^{2}\right)}
$$

For an interface under pressure forces, surface tension forces, and electric forces, the pressure term is determined by solving a stress balance for the interface. The stress balance can be solved because the curvature of the interface and surface tension coefficient are known - giving the stress from the surface tension - and the applied voltage and counter electrode separation are known - giving the electric stress. Using the equilibrium model, the position of the interface as a function of pressure difference $y=\xi(p)$ for various values of $x$ can then be expressed and compared to the position of the interface at the same points found experimentally (using MATLAB as shown in Figure 6c). By minimizing the following equation:

$$
F(p)=\sum_{j=1}^{j_{\max }}\left[\tilde{\xi}_{j}-\xi_{j}(p)\right]^{2}
$$

where $\tilde{\xi}_{j}$ are experimentally determined interface positions for given values of $x$, the pressure difference (p) across an interface can be calculated.

This technique for determining the pressure gives a pressure difference of $181 \mathrm{~Pa}$ for the interface in Figure 8a and a pressure difference of $110 \mathrm{~Pa}$ for the same interface just after the electric field is applied in Figure 8b. The application of the electric field reduces the pressure difference across the interface in static equilibrium. The pressure difference for the electrified interface in Figure $8 \mathrm{~b}$ will continue to increase after this point, as long as there is no mass loss (i.e. no electrospray), because of the continual infusion of fluid (mass) from the syringe pump.

\section{OBSERVED PHENOMENA AND APPLICATION OF THE EQUILIBRIUM MODEL}

Operational domains and images of the electrified interface are shown in Figures 10 to 13. The plotted lines are critical curves dividing equilibrium and quasi equilibrium from non equilibrium conditions. The angle with each line represents the value of the apex angle $(\theta)$ used to calculate the critical 
curve. In the operational domains, the points marked with a ' + ' represent the operating point of the interface at the instant shown in the identified figure. The letters in the operational domains correspond to the letters in the interface images, and the angles given in the interface images are the experimentally measured apex angles. The apex angle and pressure terms are determined using the technique explained in Section IV.

The experimental results show that the equilibrium model successfully predicts whether equilibrium exists and if an electrospray is formed and maintained. The operating point of all of the observed interfaces correctly sits in the equilibrium or quasi equilibrium region (to the left of the critical curve) predicted by the equilibrium model. It is possible for these points to move if the voltage or, more likely, the pressure difference across the interface changes. Movement of the operating point would be apparent by a change in the shape of the observed interface. It is clear that the magnitude of the apex angle does affect the range of operating parameters where quasi equilibrium is possible, since the critical curve moves left in the operational domain when the apex angle is increased. 

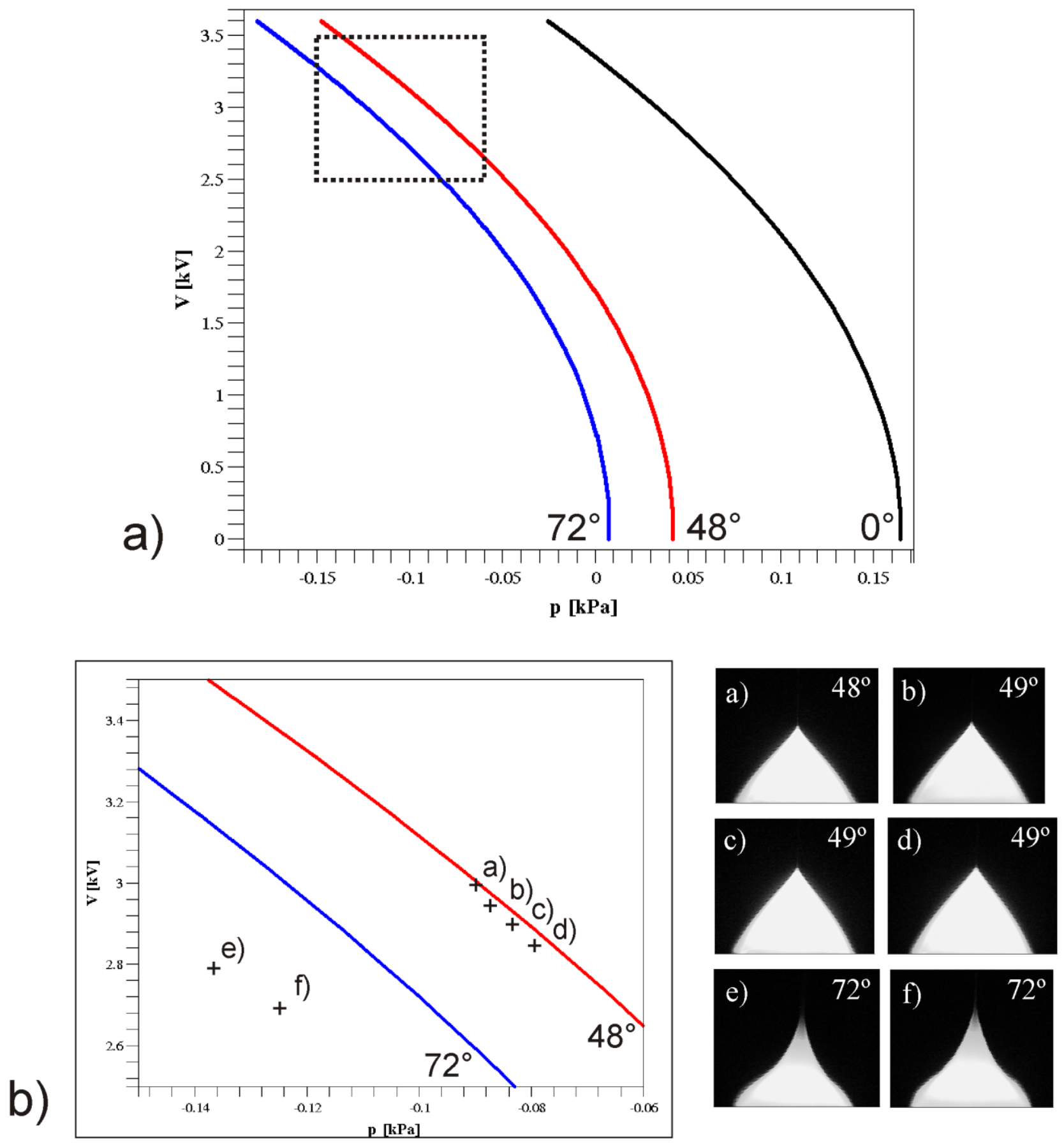

Figure 10 - a) Operational domain for counter electrode separation distance of 10mm and surface tension coefficient of $24.74 \mathrm{mN} / \mathrm{m}$. The curves, known as 'critical curves', is where the critical function equals exactly 1 for the specified apex angle. b) Zoomed in version of the operational domain. The points mark with a + represent the operating point at the instant the images of the interface are taken. 

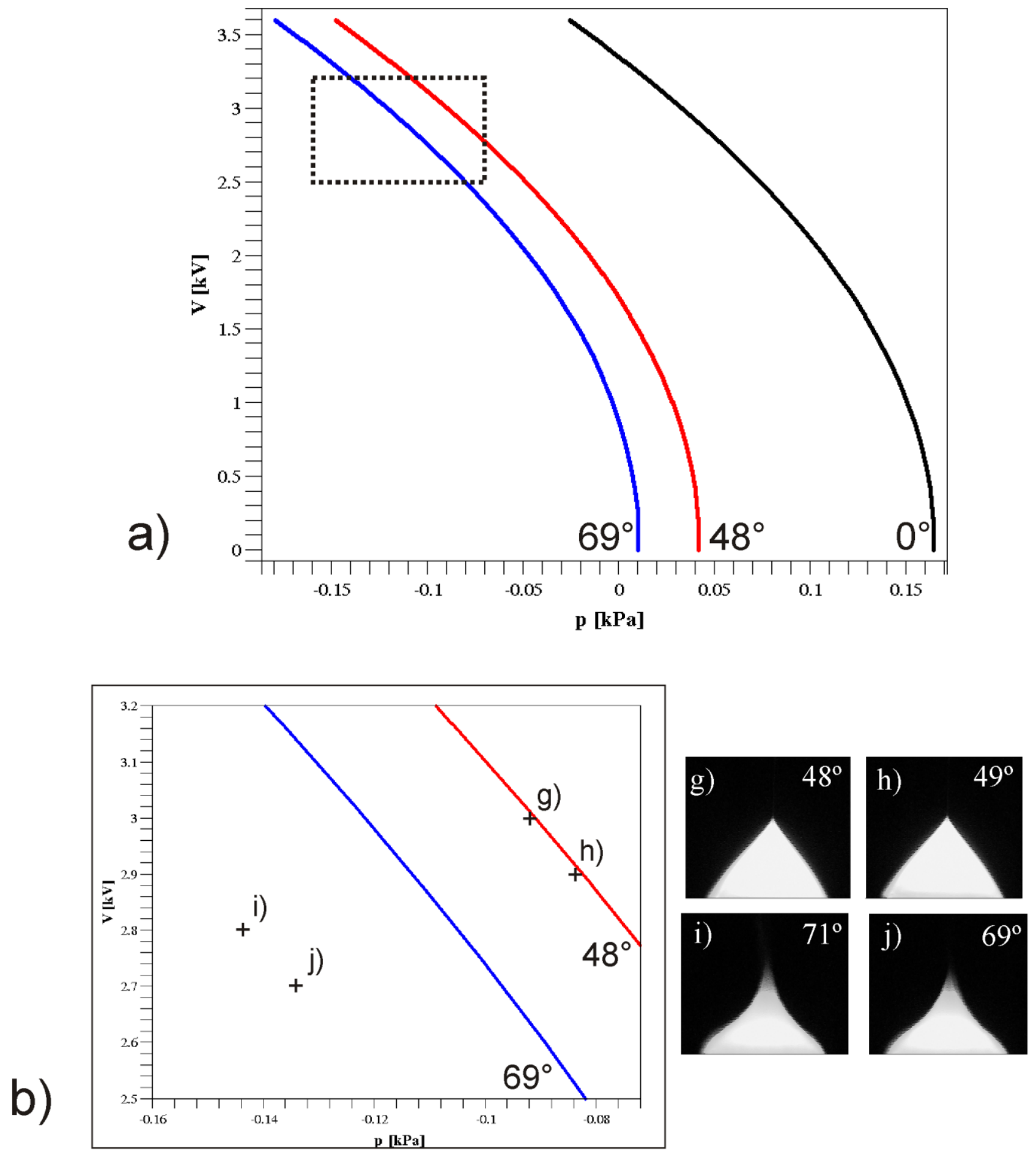

Figure 11 - a) Operational domain for a counter electrode separation distance of $10 \mathrm{~mm}$ and surface tension coefficient of $24.74 \mathrm{mN} / \mathrm{m}$. The curves, known as 'critical curves', is where the critical function equals exactly 1 for the specified apex angle. b) Zoomed in version of the operational domain. The points mark with a + represent the operating point at the instant the images of the interface are taken. 

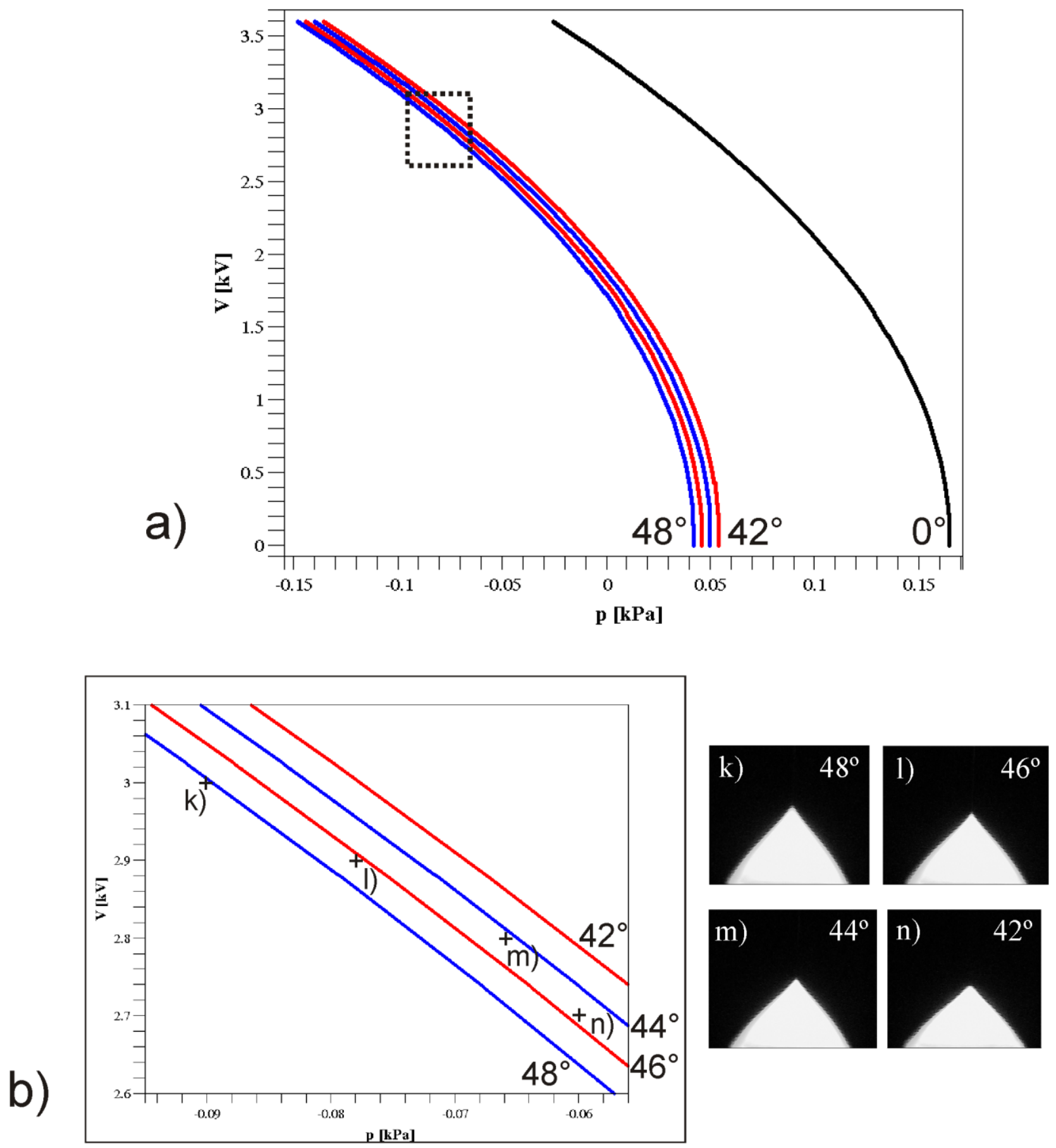

Figure 12 - a) Operational domain for a counter electrode separation distance of $10 \mathrm{~mm}$ and surface tension coefficient of $24.74 \mathrm{mN} / \mathrm{m}$. The curves, known as 'critical curves', is where the critical function equals exactly 1 for the specified apex angle. b) Zoomed in version of the operational domain. The points mark with a + represent the operating point at the instant the images of the interface are taken. 

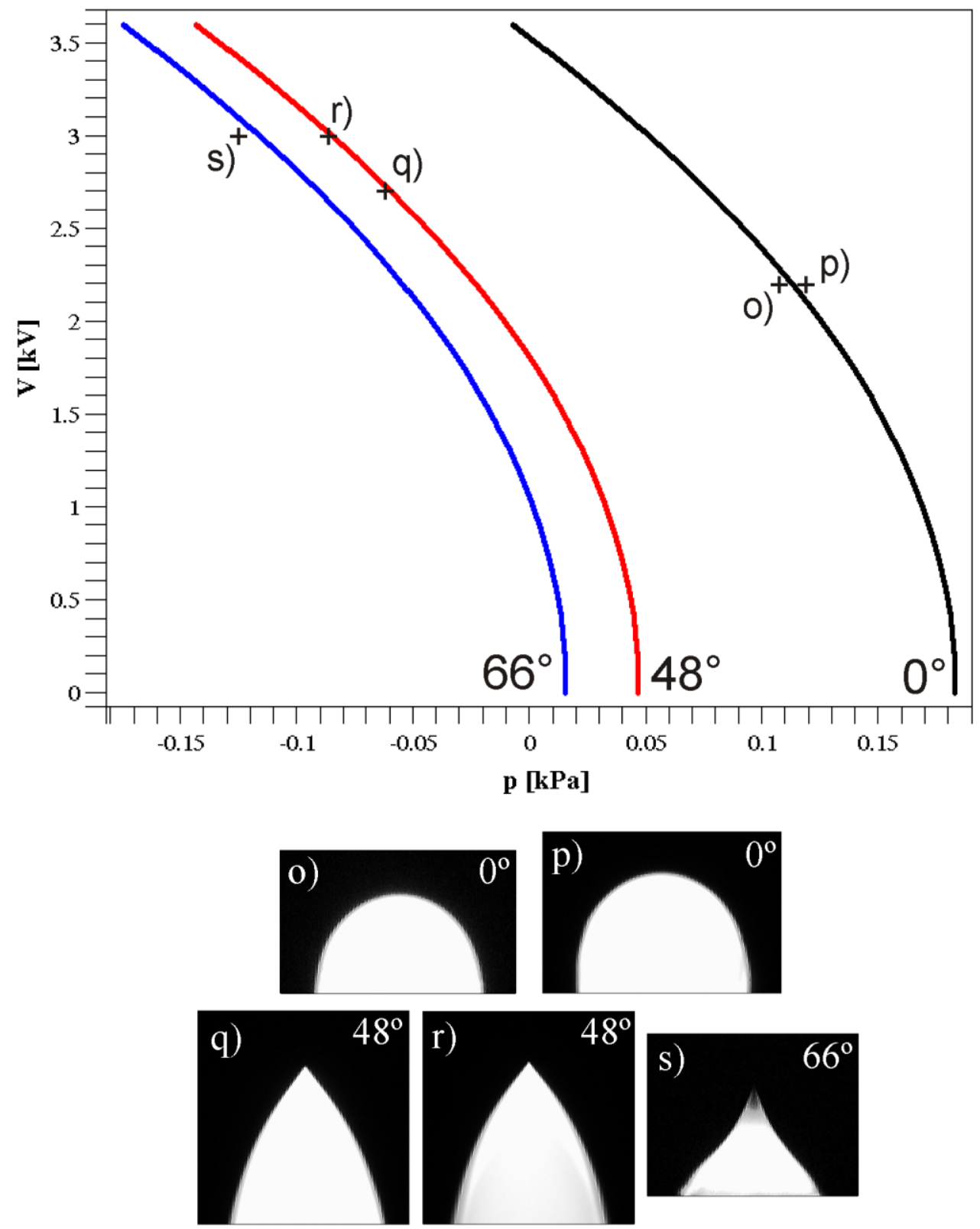

Figure 13 - Operational domain for a counter electrode separation distance of $10 \mathrm{~mm}$ and surface tension coefficient of $27.48 \mathrm{mN} / \mathrm{m}$. The curves, known as 'critical curves', is where the critical function equals exactly 1 for the specified apex angle. The points mark with a + represent the operating point at the instant the images of the interface are taken.

The interface in Figure 13(o) is in static equilibrium and not emitting a spray. The value of the critical function for this interface is less than 1 ; therefore it lies to the left of the critical curve for $s=0$ (apex angle $0^{\circ}$ ) in the operating domain shown in Figure 13. The location of this operating point indicates that a small increase in the pressure from infusion with the syringe pump will push the interface out of 
static equilibrium (i.e. the operating point will move to the right). It is found when reaching this point, the gravitational force causes the interface to be pulled down and a droplet is released from the metal tubing. This has become a non-equilibrium condition, using the definition provided by the equilibrium model, and no jet or spray has formed. Cloupeau and Prunet-Foch[23] define this as 'dripping mode'. This mode occurs at lower applied voltages and the equilibrium model is used to identify under what conditions a droplet will be released. More specifically, in this mode when the critical function exceeds 1, the droplet has become sufficiently massive that the force of gravity pulls the interface down, forming a droplet. The interface in Figure 13(p) shows the same interface in Figure 13(o) about one second later and just before a droplet is released. It can be seen that this interface now contains a massive droplet, and no spray is emitted. The location in the operational domain for this situation is identified with a ' + ' in Figure 13.

For the operational domain shown in Figure 13, it is found that the conditions shown in Figure 13(o) and Figure 13(p) mark the boundary between dripping mode where no spray is formed and other modes where sprays are formed. For all cases with $\gamma=27.48 \mathrm{mN} / \mathrm{m}$ and $\bar{h}=10 \mathrm{~mm}$, applied voltages of between 0 volts and 2200 volts (Figure 13(o)) always caused dripping mode to form after static equilibrium was broken. For the operational domains in Figures 10 to 12 , with $\gamma=24.74 \mathrm{mN} / \mathrm{m}$ and $\bar{h}$ $=10 \mathrm{~mm}$, dripping mode was formed between 0 volts and 2000 volts. This difference in voltage range is related to the surface tension coefficient: decreasing the fluid surface tension lowers the maximum voltage where dripping mode is formed.

Figure 10b(a-f), Figure 11b(g-j), Figure 12b(k-n), and Figure 13(q-s) all show interfaces in quasi equilibrium and emitting a spray. This mode of operation is most commonly used when performing electrospray and is identified by Cloupeau and Prunet-Foch[23] as 'cone-jet mode'. The operating point for these interfaces are identified in the operational domain in Figures 10 to 13, and all lie left of the critical curve for $s \neq 0$.

\section{Cone-Jet Mode}

It is clear that the shapes of the interface and apex angles are very different for the various operating conditions in Figures 10 to 13. The apex angles for the interfaces in Figure 10b(a-d), Figure 11b(g-h), Figure $12 \mathrm{~b}(\mathrm{k})$, and Figure 13(q-r) are found to be similar at approximately $48-49$ degrees. This is comparable to Taylor's apex angle of 40.7 degrees (cone semi-angle of 49.3 degrees) [12]. The apex angles in Figure 10b(e-f), Figure 11b(i-j), and Figure 13(s) are considerably larger at 66-72 degrees. Also 
noteworthy is the difference in the cone generatrix between the two cases, Figure 10b(a-d), Figure $11 \mathrm{~b}(\mathrm{~g}$ h), Figure 12b(k), and Figure 13(q-r) with a convex generatrix and Figure 10b(e-f), Figure 11b(i-j), and Figure 13(s) with a convex-concave generatrix. The ability of the interface in cone-jet mode to take on both forms was also reported by Cloupeau and Prunet-Foch, who report that the phenomenon is related to the conductivity of the fluid. However, it is important to note that for all interfaces in this study, the conductivity of the fluid was held constant.

For this study, the reason for the difference in the cone generatrix is applied voltage and pressure difference across the interface. Pantano et al. [32] have also reported the dependence of meniscus shape on pressure difference. The measured results show that the operating point of the pressure in the operational domain is related to the supplied flow rate. The impact of pressure difference (or flow rate) is best seen in Figures 13(r) and 13(s). For these two conditions, the applied voltage is held constant at 3 $\mathrm{kV}$, while the flow rate is increased from $0.3 \mu \mathrm{L} / \mathrm{min}$ in Figure 13(r) to $2 \mu \mathrm{L} / \mathrm{min}$ in Figure 13(s). The interface in Figure 13(s) is convex-concave and has a larger pressure difference (in the negative direction) compared to the interface in Figure 13(r), which is convex. As shown in the operational domain in Figure 13, the operating point for the interface in Figure 13(s) is to the left of the operating point for the interface in Figure 13(r). The experimental results indicate that larger flow rates create larger pressure differences (typically in the negative direction). Therefore, for larger flow rates, the cone generatrix tends to be convex-concave and for smaller flow rates it tends to be convex (when the applied voltage is held constant).

The impact of applied voltage on cone generatrix is shown in Figure $10 \mathrm{~b}(\mathrm{a}-\mathrm{f})$. For this situation, the flow rate is held constant at $2 \mu \mathrm{L} / \mathrm{min}$ and the voltage is reduced in steps from $3 \mathrm{kV}$ to $2.7 \mathrm{kV}$ as shown in the operational domain in Figure 10. The interface remains convex until the applied voltage is reduced to $2.775 \mathrm{kV}$ in Figure $10 \mathrm{~b}(\mathrm{e})$, when the interface becomes convex-concave. As the applied voltage is reduced from this point, the generatrix remains convex-concave. As shown in the operating domain, there is a large change in the pressure difference across the interface when the interface transforms between convex and convex-concave (note the difference in location between operating point for the interface in Figure $10 \mathrm{~b}(\mathrm{~d})$ and Figure $10 \mathrm{~b}(\mathrm{e}))$.

The equilibrium model [10] accounts for the difference in cone generatrix and is used to predict the specific pressure difference and applied voltage where the generatrix will convert from convex to convex-concave. The magnitude of this pressure difference is: 


$$
P_{\text {convex-concave }}=-\frac{\kappa}{k} T_{n n}(0, \mathbf{z})
$$

for a given applied voltage and separation distance. In Eq. 4, $T_{n n}(0, \mathbf{z})$ is the Maxwell stress at the middle of the interface $(x=0)$. A pressure difference more negative than this value will have a convex-concave interface and a pressure difference more positive than this value will have a convex interface. This value of $P_{\text {convex-concave }}$ will not exist left of the critical curve, in quasi equilibrium, for every value of the apex angle. For this reason, it is not possible to have a convex interface for a large apex angle, since the larger the apex angle, the further the critical curve is shifted to the left in the $V$ versus $P$ operational domain.

For the operating domain in Figure 10, the interface is convex-concave for the interface in Figure $10 \mathrm{~b}(\mathrm{e})$, corresponding to an applied voltage of $2.775 \mathrm{kV}$ and pressure of $-13.5 \times 10^{-4}$ (or $-137 \mathrm{~Pa}$ ). Using the equilibrium model, the value of $P_{\text {convex-concave }}$ for an applied voltage of $2.775 \mathrm{kV}$ and electrode separation of $10 \mathrm{~mm}$ is calculated to be $-4.6 \times 10^{-4}$. Since the value of the pressure for the interface in

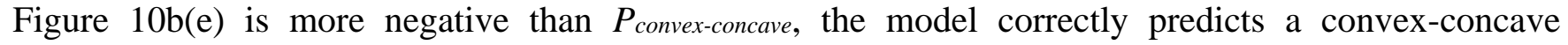
generatrix.

The equilibrium model does allow, at very large negative pressure differences, for the interface to be fully concave. Fully concave interfaces has been reported in the literature - specifically when dealing with liquid metal ion sources [33] - however we were unable to produce this interface shape using the fluid in this study.

The operating domains in Figures 10 to 13 show that the operating points of the fully convex interfaces lie very close to the critical curve, while the convex-concave interfaces lie further to the left of the critical curve. This implies that the fully convex interface is very close to the boundary of equilibrium while the convex-concave interface is not. This was experimentally supported since during testing it was often difficult to maintain interfaces with fully convex shapes, as small disturbances or fluctuations in pressure would cause the interface to break apart. This was not the cases for the convex-concave interface, which would maintain its shape even during small disturbances. This result is consistent with the location of the operating points in the operating domains in Figures 10 to 13. Small disturbances or small changes in pressure will cause interfaces close to the boundary of equilibrium to break apart easily, while interfaces further from the boundary of equilibrium can be more easily maintained. 


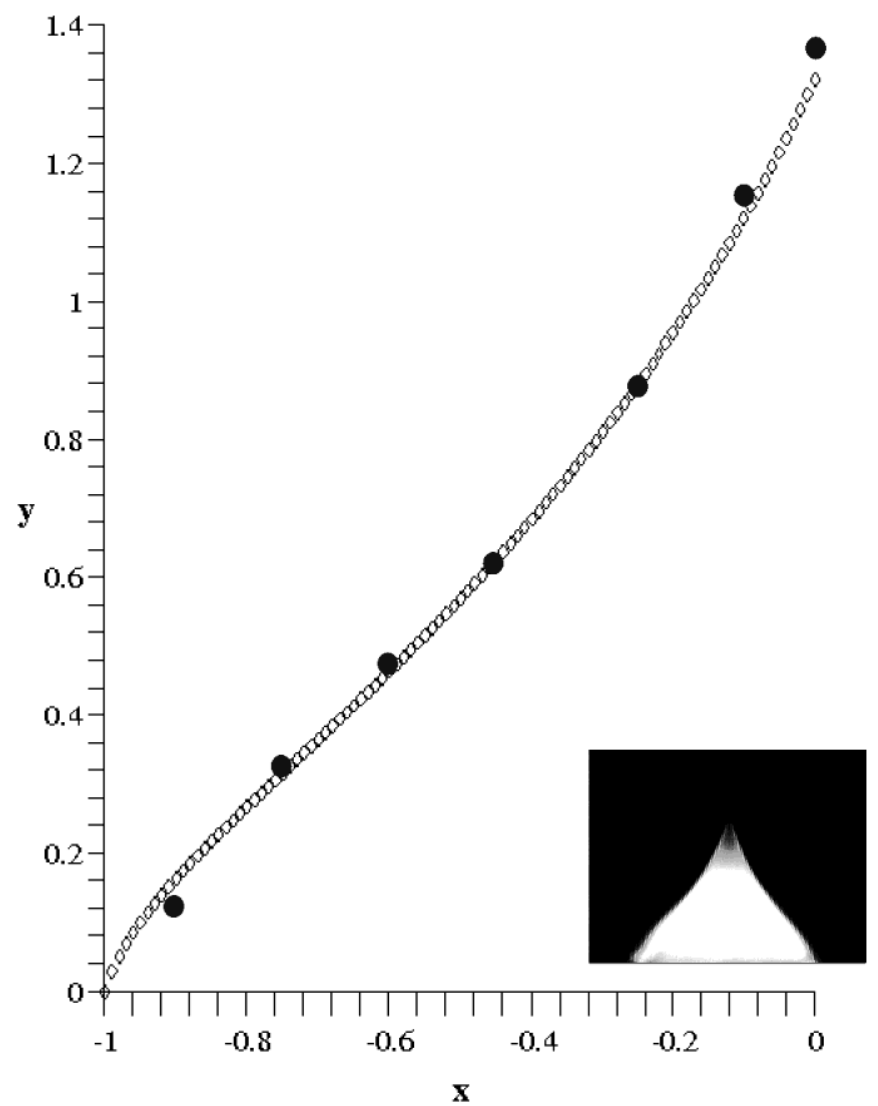

Figure 14 - Predicted shape of the interface in Figure 13(s) using the equilibrium model. The actual image of the interface is shown again at the inset. The scales are made non-dimensional using the characteristic length of $L_{\varepsilon}=150$ $\mu \mathrm{m}$. The filled black circles represent measured points on the actual interface. The open circles are calculated points using the equilibrium model. Symmetry is assumed so only half of the interface is plotted.

\section{Interface Shape and Tip Height}

The equilibrium model is able to predict the shape of the electrified interface under the three stresses considered in this study. The shape predicted using the equilibrium model for the convex-concave interface in Figure 13(s) is shown in Figure 14. The model is able to predict with very good accuracy the shape of the interface. The measured tip height was found to be 1.367 and the predicted tip height using the equilibrium model was 1.323 - a difference of about 3\% (note that the heights are made nondimensional using the characteristic length $L_{\varepsilon}=150 \mu \mathrm{m}$ (see Figure 7)). All points on the interface remain steady and unchanged once the final quasi equilibrium state is attained, with the exception of points near the apex of the cone. These points can fluctuate slightly in the y-direction during steady operation, and this may account for the small discrepancies in tip height between the model and experimental results. 
The ability to predict the shape of the interface is important for two reasons. First, from a component design perspective, it can be useful to know the height of the interface during electrospray. Second, the description of the electric field can be improved if the shape of the interface is known; leading to improved modeling.

Reznik et al.[19] addressed tip height of an electrified interface up to the limit of jet formation. Using the equilibrium model and experimental results, the impact of the operational parameters on the tip height during quasi-equilibrium (during jetting) can be determined here. The interfaces in Figure 10(a-f) are taken for decreasing applied voltage and a constant flow rate. For the convex interfaces in Figure 10(a-d), the interface tip height is increasing along with the increasing pressure. The tip height also increases when moving from the interface in Figure $10 \mathrm{~b}(\mathrm{e})$ to Figure $10 \mathrm{~b}(\mathrm{f})$. The tip heights of the interfaces in Figure 13(q-r) are significantly higher than that of the interfaces in Figure 10(a-d). This corresponds to a higher surface tension coefficient for the interfaces in Figure 13(q-r) compared to the interface in Figure 10(a-d). Note that the tip height of the interfaces in Figure 10b(a) and Figure 11b(g) are similar because the applied voltage and pressure difference are equal between these two conditions. The experimental results show the quasi equilibrium tip height will increase when: (1) the pressure is increased in the positive direction or (2) the surface tension coefficient is increased. This result confirms findings from the equilibrium model [10].

There is one requirement that needs to be added to the tip height dependence on pressure difference and surface tension coefficient. It can be seen that for the interfaces in Figure $12 b(k-n)$, the tip height decreases with increasing pressure. However, it can be observed that the magnitude of the apex angle is also changing for the interfaces in Figure $12 \mathrm{~b}(\mathrm{k}-\mathrm{n})$. This indicates that the increase in tip height with increasing pressure and surface tension coefficient is only valid for a constant apex angle. This can be explained using the equilibrium model: the parameter $s$ affects interface equilibrium along with the pressure difference and electric stress (Eq. 1). Decreasing the magnitude of the apex angle means the pressure difference (which is negative in this case) can move in the positive direction while still maintaining equilibrium. This is combined with the fact that the tip height must decrease with decreasing apex angle to maintain the fully convex interface for the interfaces in Figure $12 b(k-n)$ (details on interface generatrix are given in the previous subsection).

It is interesting to note that the interface in Figure $12 \mathrm{~b}(\mathrm{n})$ has an apex angle that is closest to that predicted by Taylor. In Figure $12 \mathrm{~b}(\mathrm{n})$, the apex angle is $42^{\circ}$ (a Taylor angle of $48^{\circ}$ ). The magnitude of the operating conditions used in Figure $12 \mathrm{~b}(\mathrm{n})$ are the smallest compared to all the other operating 
conditions used for the other interfaces in this study. The applied voltage is $2.7 \mathrm{kV}$ and the pressure difference is $-60 \mathrm{~Pa}$. This indicates that reducing the electric stress and surface tension forces on the interface, while maintaining quasi equilibrium, moves the magnitude of the apex angle closer to the result predicted by Taylor.

\section{Operational Domain for a Fixed Pressure Condition}

As mentioned above, it is possible to fix the value of the applied pressure so that the impact of applied voltage and electrode separation distance can be considered. This operating domain is shown in Figure 15, and it is again divided into two subdomains where equilibrium can and can not exist.

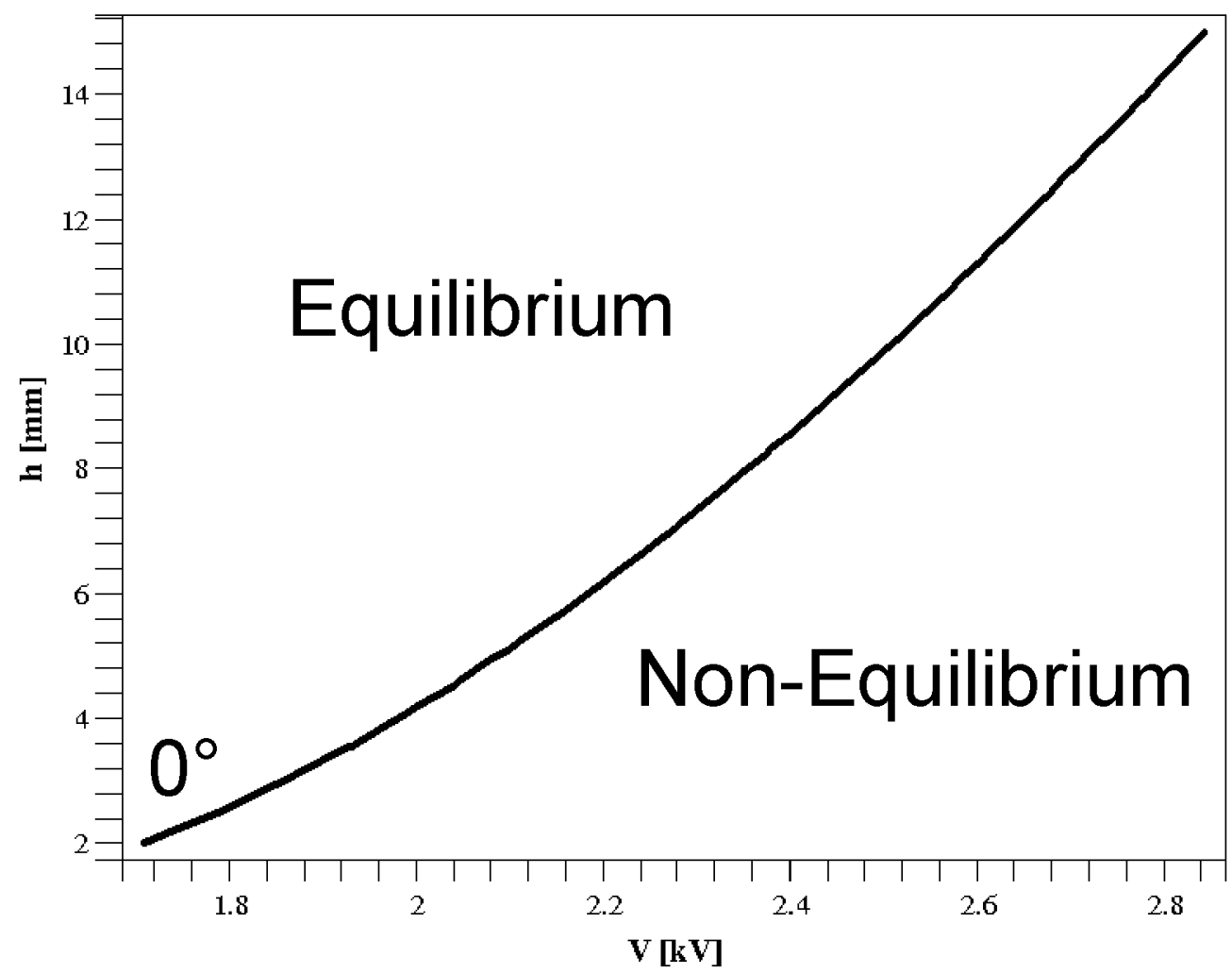

Figure 15 - Operational domain for $\mathrm{p}=0.0916 \mathrm{kPa}$ - half the maximum positive pressure for this fluid. The curve represents where the critical function equals exactly 1.

The line dividing the plane is where the critical function is equal to 1 for $s=0$ (the apex angle is $\left.0^{\circ}\right)$. The pressure is fixed at half the value of the maximum possible positive pressure difference for this fluid and interface size. Points that would be left of the critical curve are in static equilibrium. 


\section{Structure of the Operational Domain}

The domains shown in Figures 10-13 and 15 are useful to determine the required operating conditions that are necessary to break static equilibrium and, with sufficient applied voltage, form and maintain an electrospray. Three key parameters are considered - applied voltage, electrode separation, and pressure difference (also sine of the angle at the apex) - and the operational domain predicts the transition point from a static equilibrium to quasi equilibrium state, and the conditions necessary to maintain an electrospray.

The transition point between 'dripping mode' and 'cone-jet mode' is less well defined, and this point is determined empirically. For the tube geometry considered here and a separation distance of $10 \mathrm{~mm}$, the transition was at 2000 volts for $\gamma=24.74 \mathrm{mN} / \mathrm{m}$ and 2200 volts for $\gamma=27.48 \mathrm{mN} / \mathrm{m}$. However, depending on the geometry and working fluid, it is possible for this value to be even lower. Arscott and Troadec [34] have reported the onset of electrospray at voltages as low as 125 volts in a nanometer sized conduit. Reznik et al.[19] and Harris and Basaran [14] have investigated the onset of jetting (i.e. the onset of cone-jet mode), however their work dealt specifically with isolated droplets. It is interesting to note that Reznik et al.[19] found a critical electrical Bond number (ВоE) of about 3.04 for a contact angle of $\pi / 2$ for the isolated droplet (the electrical Bond number compares the relative importance of electrical stress and capillary stress in a similar manner to the non-dimensional parameter $\kappa$ in the critical function). This value from Reznik et al. is comparable to measured electrical Bond numbers of 3.62 and 3.94 for the transition between 'dripping mode' and 'cone-jet mode' for $\gamma=24.74 \mathrm{mN} / \mathrm{m}$ and $\gamma=27.48 \mathrm{mN} / \mathrm{m}$, respectively. These values for Boe are determined using Eq. 5 (from [19]) and the electric field at $x=0$ from the equilibrium model [10].

$$
B o_{E}=\frac{L_{\varepsilon} E^{2}}{\gamma}
$$

Eq. 5 is expressed for CGS units (same as [19]) using the nomenclature from this study: $L_{\varepsilon}$ is the characteristic length, $E$ is the electric field at $x=0$, and $\gamma$ is the surface tension coefficient. 


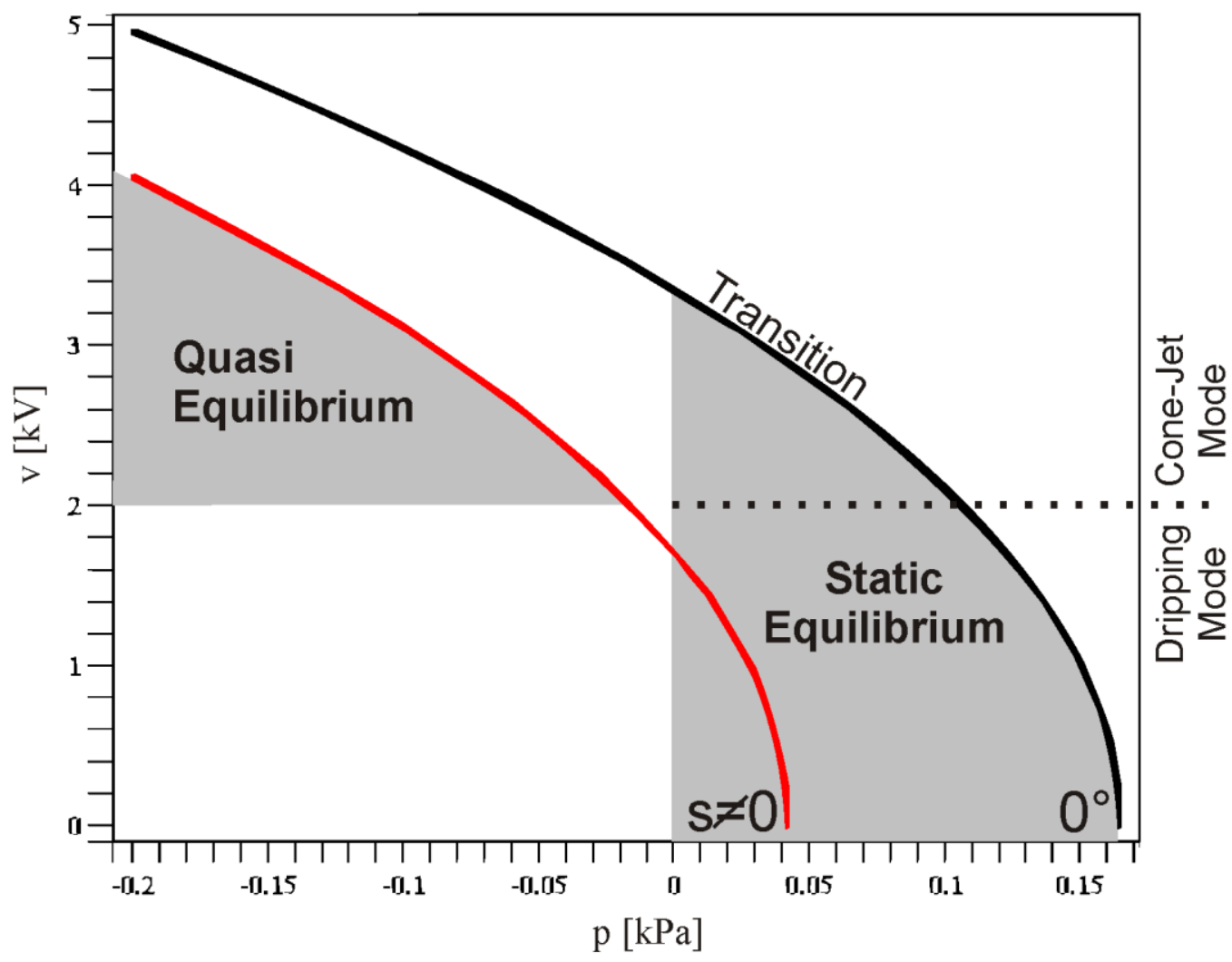

Figure 16 - Operational domain for an electrode separation distance of $10 \mathrm{~mm}$ and surface tension coefficient of 24.74 $\mathrm{mN} / \mathrm{m}$. The important regions of operation are identified and have been confirmed experimentally. The critical curve marked as 'Transition' represents the transition from static equilibrium to quasi equilibrium in cone jet mode or the

formation of a droplet in dripping mode. The line dividing cone-jet mode and dripping mode is determined empirically. The unmarked regions in white represent non-equilibrium regions or are regions that can not be attained.

The operating domains can be extended to identify the important regions of operation. The regions of operation for a constant electrode separation distance of $10 \mathrm{~mm}$ are shown in Figure 16. Figure 16 is the same operating domain as Figure 10, but with critical curves for only $s=0$ and one $s \neq 0$ value. The location of the critical curve for $s \neq 0$ will vary depending on the magnitude of the apex angle, however the critical curve for $s=0$ will remain unchanged for a given interface and counter electrode width.

In Figure 16, it is important to note that the static equilibrium region only exists when $s=0$, it does not exist when $s \neq 0$. The entire region to the right of the critical curve for $s \neq 0$ is a non-equilibrium region. The region to the left of the critical curve for $s \neq 0$ and below the quasi equilibrium region can not be attained, as points that fall below the quasi equilibrium region will have an $s=0$, and move into the static equilibrium region. The lower boundary of the quasi equilibrium region is defined by the boundary between cone-jet mode and dripping mode. 
The ability to predict the conditions necessary to break static equilibrium and form and maintain an electrospray allows better design decision to be made, since it is useful to apply only the minimum required electric field (a function of voltage and separation distance) and pressure difference; and to avoid excessive magnitudes. This will minimize the power requirements, the geometric constraints on channel size, and the mechanical stresses on the emitter device.

\section{CONCLUSIONS}

An experimental investigation has been performed to investigate the properties of an electrified fluid interface and the formation of an electrospray. The results are related to a plane model of fluid interface rupture in an electric field previously developed by Gubarenko et al. [10]. The experimental results revealed that the equilibrium model accurately predicts when the interface will be in equilibrium and the necessary operational conditions to form and maintain an electrospray. This study - coupled with the equilibrium model - revealed important properties of electrified interfaces, including generatrix and tip height (i.e. shape) dependence on operational parameters, impact of apex angle on equilibrium, dripping mode and cone-jet mode transition, and structure of operational domains.

Testing was performed using an electrospray emitter chip fabricated using Polydimethylsiloxane (PDMS) and glass. This prototype allowed for a stable interface to be formed in cone-jet mode, the most common operating mode. The prototype is uncomplicated to fabricate, has limited dead volumes, and it is designed to be a platform where electrospray can be combined with upstream sampling processing.

For applications where electrified fluid interfaces are used, such as mass spectrometry, material deposition, and colloid thrusters for propulsion, it is important to understand what operating conditions lead to the onset and steadiness of a quasi equilibrium state, specifically electrospray. It is useful to apply only the minimum required electric field (a function of voltage and separation distance) and pressure to form and maintain an electrospray, and to avoid excessively large voltages and pressures. This will minimize the power requirements, the geometric constraints on channel size, and the mechanical stresses on the emitter device. The equilibrium model and the experimental results reported here contribute to the understanding of electrified fluid interface phenomenon.

\section{ACKNOWLEDGMENTS}

The authors gratefully acknowledge the financial support of the Natural Sciences and Engineering 
P. R. Chiarot, S. I. Gubarenko, R. Ben Mrad, and P. Sullivan, "Application of an Equilibrium Model for an Electrified Fluid InterfaceElectrospray Using a PDMS Microfluidic Device,” J. Microelectromechanical Syst., vol. 17, no. 6, pp. 1362-1375, 2008.

10.1109/JMEMS.2008.2006822

Research Council (NSERC) of Canada and Engineering Services Inc. (ESI).

\section{REFERENCES}

[1] W. Balachandran, P. Miao, and P. Xiao, "Electrospray of Fine Droplets of Ceramic Suspensions for Thin-Film Preparation,” J. Electrostat., vol. 50, no. 4, pp. 249-263, Mar. 2001.

[2] C. Satterley, L. Perdigao, A. Saywell, G. Magnano, A. Rienzo, L. Mayor, V. Dhanak, P. Beton, and J. O'Shea, "Electrospray Deposition of Fullerenes in Ultra-high Vacuum: In Situ Scanning Tunneling Microscopy and Photoemission Spectroscopy," Nanotechnology, vol. 18, 455304, Jan. 2007.

[3] M. Gamero-Castano and V. Hruby, "Electrospray as a Source of Nanoparticles for Efficient Colloid Thrusters,” J. Propul. Power, vol. 17, no. 5, pp. 977-987, Sept. 2001.

[4] J.B. Fenn, M. Mann, C.K. Meng, S.F. Wong, and C.M. Whitehouse, "Electrospray Ionization for Mass Spectrometry of Large Biomolecules,” Science, vol. 246, no. 4926, pp. 64-71, Oct. 1989.

[5] R.S. Ramsey and J.M. Ramsey, "Generating Electrospray from Microchip Devices Using Electroosmotic Pumping,” Anal. Chem., vol. 69, pp. 1174-1178, Mar. 1997.

[6] L. Licklider, X. Wang, A. Desai, Y. Tai, and T. Lee, "A Micromachined Chip-Based Electrospray Source for Mass Spectrometry,” Anal. Chem., vol. 72, pp. 367-375, Jan. 2000.

[7] S. Le Gac, S. Arscott, and C. Rolando, "A Planar Microfabricated Nanoelectrospray Emitter Tip Based on a Capillary Slot," Electrophoresis, vol. 24, pp. 3640-3647, Nov. 2003.

[8] M. Bedair and R. Oleschuk, "Fabrication of Porous Polymer Monoliths in Polymeric Microfluidic Chips as an Electrospray Emitter for Direct Coupling to Mass Spectrometry," Anal. Chem., vol. 78, pp. 1130-1138, Feb. 2006.

[9] S. Koster and E. Verpoorte, "A Decade of Microfluidic Analysis Coupled with Electrospray Mass Spectrometry: An Overview," Lab Chip, vol. 7, pp. 1394-1412, Sept. 2007.

[10] S. Gubarenko, P. Chiarot, R. Ben Mrad, and P. Sullivan, "Plane Model of Fluid Interface Rupture in an Electric Field," Phys. Fluids, vol. 20, no. 4, 043601, Apr. 2008.

[11] J. Zeleny, "Instability of Electrified Liquid Surfaces," Phys. Rev., vol. 10, no. 1, pp. 1-6, 1917.

[12] G. I. Taylor, "Disintegration of Water Drops in Electric Field," Proc. R. Soc. Lond., vol. 280, no. 1382, pp. 383-397, Jul. 1964.

[13] O. Basaran and L. E. Scriven, "Axisymmetric Shapes and Stability of Pendant and Sessile Drops in an Electric Field," J. Colloid Interf. Sci., vol. 140, no. 1, pp. 10-30, Nov. 1990.

[14] M. Harris and O. Basaran, "Capillary Electrohydrostatics of Conducting Drops Hanging from a Nozzle in an Electric Field,” J. Colloid Interf. Sci., vol. 161, pp. 389-413, Dec. 1993.

[15] F. Wohlhuter and O. Basaran, "Shapes and Stability of Pendant and Sessile Dielectric Drops in an Electric Field," J. Fluid Mech., vol. 235, pp. 481-510, Feb. 1992.

[16] H. Stone, J. Lister, and M. Brenner, "Drops with Conical Ends in Electric and Magnetic Fields," Proc. R. Soc. Lond. A, vol. 455, pp. 329-347, Jan. 1999.

[17] L. Cherney, "Structure of Taylor Cone-jets: Limit of Low Flow Rates," J. Fluid Mech., vol. 378, pp. 167-196, Jan. 1999.

[18] N. Sujatha, P.H. Cutler, E. Kazes, J.P. Rogers, and N.M. Miskovsky, "Variational Formulation for the Equilibrium Condition of a Conducting Fluid in an Electric Field," Appl. Phys. A, vol. 32, pp. 55-61, Oct. 1983. 
[19] S. Reznik, A. Yarin, A. Theron, and E. Zussman, "Transient and Steady Shapes of Droplets Attached to a Surface in a Strong Electric Field," J. Fluid Mech., vol. 516, pp. 349-377, Sept. 2004.

[20] J. Fernandez de la Mora, "The Effect of Charge Emission from Electrified Liquid Cones," J. Fluid Mech., vol. 243, pp. 561-574, Oct. 1992.

[21] J. Fernandez de la Mora and I. G. Loscertales, "The Current Emitted by Highly Conducting Taylor Cones," J. Fluid Mech., vol. 260, pp. 155-184, Feb. 1994.

[22] A. M. Ganan-Calvo, J. Davila, and A. Barrero, "Current and Droplet Size in the Electrospraying of Liquids. Scaling Laws,” J. Aerosol Sci., vol. 28, no. 2, pp. 249-275, Mar. 1997.

[23] M. Cloupeau and B. Prunet-Foch, "Electrohydrodynamic Spraying Functioning Modes: A Critical Review," J. Aerosol Sci., vol. 25, no. 6, pp. 1021-1036, Sept. 1994.

[24] J. Fernandez de la Mora, "The Fluid Dynamics of Taylor Cones," Annu. Rev. Fluid Mech. vol. 39, pp 217-243, Jan. 2007.

[25] S. Arscott, S. Le Gac, and C. Rolando, "A Polysilicon Nanoelectrospray-Mass Spectrometry Source Based on a Microfluidic Capillary Slot," Sensor. Actuat. B, vol. 106, pp. 741-749, May 2005.

[26] D. Duffy, J. McDonald, O. Schueller, and G. Whitesides, "Rapid Prototyping of Microfluidic Systems in Poly(dimethylsiloxane)," Anal. Chem., vol. 70, pp. 4974-4984, Dec. 1998.

[27] B. Jo, L. Van Lererghe, K. Motsegood, and David Beebe, "Three-Dimensional Micro-Channel Fabrication in Polydimethylsiloxane (PDMS) Elastomer," J. Microelectromech. Syst., vol. 9, no. 1, pp. 76-81, Mar. 2000.

[28] C. Effenhauser, G. Bruin, and A. Paulus, "Integrated chip-based capillary electrophoresis," Electrophoresis, vol. 18, no. 12-13, pp. 2203-2213, Nov. 1997.

[29] Q. Xue, F. Foret, Y. Dunayevskiy, P. Zavracky, N. McGruer, and B. Karger, "Multichannel Microchip Electrospray Mass Spectrometry," Anal. Chem., vol. 69, pp. 426-430, Feb. 1997.

[30] D. Lide (editor), CRC Handbook of Chemistry and Physics. 87 ${ }^{\text {th }}$ Edition, Online Resource, 2006.

[31] J. Eggers, "Nonlinear Dynamics and Breakup of Free-Surface Flows," Rev. Mod. Phys., vol. 69, no. 3, pp. 865-929, Jul. 1997.

[32] C. Pantano, A. M. Ganan-Calvo, and A. Barrero, "Zeroth-order, Electrohydrostatic Solution for Electrospraying in Cone-jet Mode," J. Aerosol Sci., vol. 25, pp. 1065-1077, Sept. 1994.

[33] V. Suvorov and N. Zubarev, "Formation of the Taylor Cone on the Surface of Liquid Metal in the Presence of an Electric Field,” J. Phys. D: Appl. Phys., vol. 37, pp. 289-297, Jan. 2004.

[34] S. Arscott and D. Troadec, "Electrospraying from Nanofluidic Capillary Slot," Appl. Phys. Lett., vol. 87, no. 134101, Sept. 2005. 